\title{
The Anharmonic Ratios of 4 points in a straight line.
}

\author{
By R. F. Muirhead, D.Sc.
}

(Read 12th November 1909. Received 14th January 1910).

The anharmonic ratio of four points A, B, C, D depends on the order in which they are taken. The A.I.G.T. "Syllabus," following Cremona, defines the symbol (ABCD) to stand for $\frac{\mathrm{AC}}{\mathrm{BC}}: \frac{\mathrm{AD}}{\mathrm{BD}}$, when $\mathrm{A}$ is conjugate to $\mathrm{B}$ and $\mathrm{C}$ to $\mathrm{D}$. Thus, taking $\lambda$ to denote the value of this double-ratio, we have

$$
(\mathrm{A}, \mathrm{B}, \mathrm{C}, \mathrm{D})=\frac{\mathrm{AC}}{\mathrm{BC}}: \frac{\mathrm{AD}}{\mathrm{BD}}=\frac{\mathrm{AC}}{\mathrm{CB}} \cdot \frac{\mathrm{BD}}{\mathrm{DA}}=\lambda \text {. }
$$

There are $4 !=24$ different orders in which 4 letters may be taken, but these do not give rise to 24 but only to 6 different values of the anharmonic ratio.

This may be demonstrated in different ways. One method is to take the anharmonic ratio in the form $\frac{\mathrm{AC} . \mathrm{BD}}{\mathrm{CB} . \mathrm{DA}}$ and observe that the numerator and the denominator each give the area of a rectangle contained by two non-coterminous segments of the line. Now it is obvious that there are only 3 rectangles of that sort (if we do not take account of the sign of the segments), viz., $p \equiv \mathrm{AB}$. CD, $q \equiv \mathrm{AC}$. DB, $r \equiv \mathrm{AD}$. BC. Hence, so far as absolute magnitude is concerned, there are only 6 different values of the anharmonic ratio, which may be written $\frac{p}{q}, \frac{q}{p}, \frac{p}{r}, \frac{r}{\rho}, \frac{q}{r}, \frac{r}{q}$; and it is easy to verify that the algebraic values are got by prefixing the negative sign to each of these quantities; or we can see that it must be so since in the form $\frac{\mathrm{AC} . \mathrm{BD}}{\mathrm{CB} . \mathrm{DA}}$ each letter begins one and only one segment, whereas if we take two of the rectangles $p, q, r$, $A$ and $A$ only begins two segments. It is well known that if the 
value of one of these anharmonic ratios be denoted by $\lambda$, those of the others are given by $\frac{1}{\lambda}, 1-\lambda, \frac{1}{1-\lambda}, \frac{\lambda}{\lambda-1}$ and $\frac{\lambda-1}{\lambda}$.

This may be shown by using the identity $p+q+r=0$.

To prove the identity we observe that

$$
\begin{aligned}
\mathrm{AB} \cdot \mathrm{CD} & +\mathrm{AC} \cdot \mathrm{DB}+\mathrm{AD} \cdot \mathrm{BC}=\mathrm{AB} \cdot \mathrm{CD}+(\mathrm{AB}+\mathrm{BC}) \mathrm{DB}+\mathrm{AD} \cdot \mathrm{BC} \\
& =\mathrm{AB}(\mathrm{CD}+\mathrm{DB})+\mathrm{BC}(\mathrm{AD}+\mathrm{DB})=\mathrm{AB} \cdot \mathrm{CB}+\mathrm{BC} \cdot \mathrm{AB} \\
& =\mathrm{AB}(\mathrm{CB}+\mathrm{BC})=\mathrm{AB} \cdot \mathrm{CC}=0 .
\end{aligned}
$$

Dividing the identity by $-p$ we have

$$
-1-\frac{q}{p}-\frac{r}{p}=0 \text {. }
$$

Thus if we denote by $\lambda$ and $\lambda^{\prime}$ the anharmonic ratios $-\frac{q}{p}$ and $-\frac{r}{p}$ we have

$$
\lambda+\lambda^{\prime}=1 \text {. }
$$

Since $-\frac{q}{p}$ and $-\frac{r}{p}$ are also reciprocals of anharmonic ratios, we see that we have also pairs $\lambda \lambda^{\prime}$ related in the following ways :

$$
\begin{aligned}
& \lambda+\frac{1}{\lambda^{\prime}}=1 . \\
& \frac{1}{\lambda}+\frac{1}{\lambda^{\prime}}=1 .
\end{aligned}
$$

(i) and (iii) give $\lambda^{\prime}=1-\lambda$ and $\lambda^{\prime}=\frac{1}{\lambda-1}$, and are not altered by interchanging $\lambda$ and $\lambda^{\prime}$. (ii) gives $\lambda^{\prime}=\frac{1}{1-\lambda}$, and by interchanging. $\lambda$ and $\lambda^{\prime}$ we get $\lambda^{\prime}=\frac{\lambda-1}{\lambda}$.

The remaining case is when one ratio is the reciprocal of another.

It is perhaps worth noting that to get the relation between any two anharmonic ratios of 4 points which are not simply reciprocal, for example, $\lambda=\frac{\mathrm{AC} \cdot \mathrm{BD}}{\overline{\mathrm{CB}} \cdot \mathrm{DA}}$ and $\lambda^{\prime}=\frac{\mathrm{BA} \cdot \mathrm{DC}}{\mathrm{AD} \cdot \mathrm{CB}}$, we have only to observe which rectangle (in this case $A D$. CB) occurs in both, and then to divide by it the identical relation

$$
\mathrm{AB} \cdot \mathrm{CD}+\mathrm{AC} \cdot \mathrm{DB}+\mathrm{AD} \cdot \mathrm{BC}=0 \text {, }
$$

whence $\lambda^{\prime}+\lambda-1=0$ or $\lambda^{\prime}=1-\lambda$. 
The six values $\lambda, \frac{1}{\lambda}, 1-\lambda, \frac{1}{1-\lambda}, \frac{\lambda}{\lambda-1}, \frac{\lambda-1}{\lambda}$ may be arranged as follows, after the manner suggested by $\mathrm{Mr}$ Alison's Mnemonic for Trigonometrical Ratios given in Vol. IV. of these Proceedings.

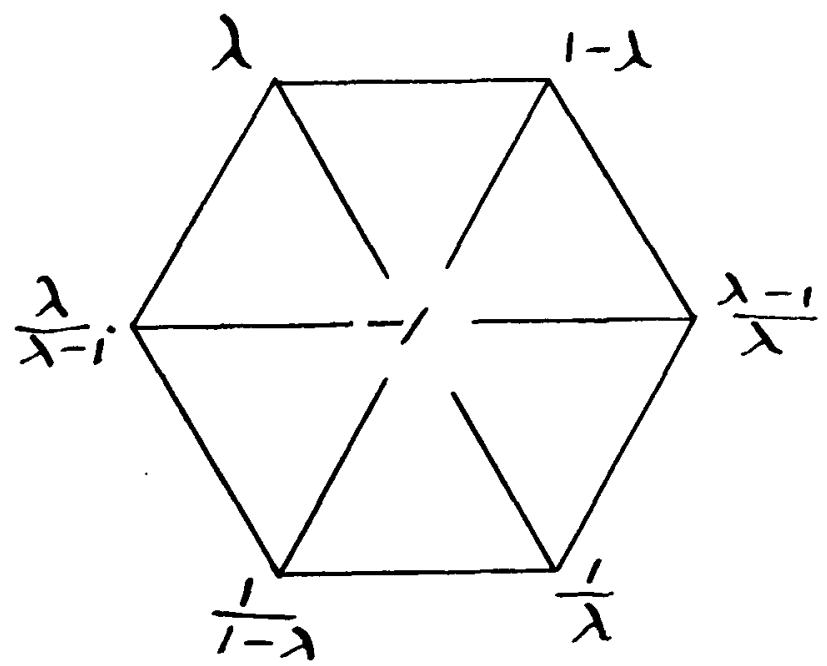

Fig. 1.

Here the following rules hold good:

(1) Taking any three successive quantities, either across the diameter or round the circumference, the first is equal to the ratio of the second to the third taken negatively.

(2) The sum of the quantities at the three corners of any downward pointing triangle is $=0$.

(3) The sum of the reciprocals of those at the corners of any upward pointing triangle $=0$.

(4) The product of the quantities at either set of three alternate corners of the hexagon $=-1$.

The connection between this and Mr Alison's figure is brought out if we put $\lambda=\sin ^{2} \theta$, then the other quantities taken in order round the diagram are $\cos ^{2} \theta,-\cot ^{2} \theta, \operatorname{cosec}^{2} \theta, \sec ^{2} \theta,-\tan ^{2} \theta$. 
Of course, that $\theta$ may be real $\lambda$ must not lie outside the interval 0 to 1 , and that one at least of the anharmonic ratios of four points satisfies this condition is obvious.

The geometric interpretation of $\theta$ is as follows :

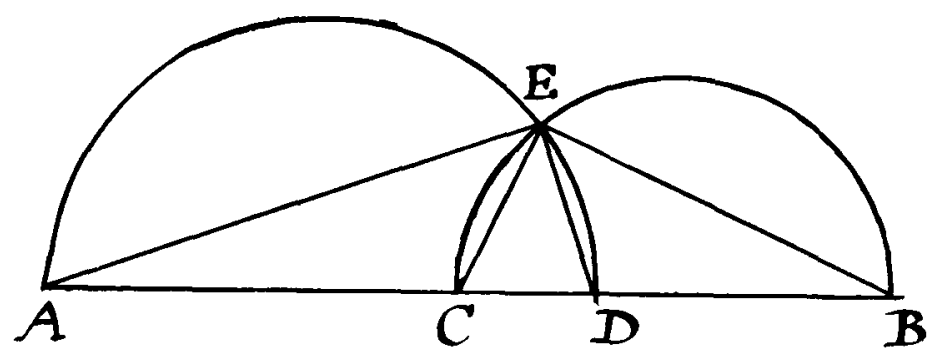

Fig. 2.

Let circles be described on those segments as diameters which only partly overlap (in the figure, $A D$ and $C B$ ) and let the circles intersect at $\mathbf{E}$.

Then denoting the equal angles $A E C, D E B$ by $\theta$, we have

$$
\begin{aligned}
\frac{\mathrm{AC} \cdot \mathrm{BD}}{\mathrm{CB} \cdot \mathrm{DA}} & =\frac{\triangle \mathrm{ACE}}{\triangle \mathrm{CBE}} \cdot \frac{\triangle \mathrm{BDE}}{\triangle \mathrm{DAE}}=\frac{\mathrm{AE} \cdot \mathrm{CE} \sin \theta}{\mathrm{CE} \cdot \overline{\mathrm{BE}}} \cdot \frac{\mathrm{BE} \cdot \mathrm{DE} \sin \theta}{\mathrm{DE} \cdot \mathrm{AE}} \\
& =\sin ^{2} \theta .
\end{aligned}
$$

[This construction I find is given in Casey's "Conics" on page 66 of the lst edition.]

Now it has often occurred to me that (ABCD) could be with advantage written in square array thus: $\left\{\begin{array}{l}A D \\ C B\end{array}\right\}$, so that the conjugate points $A B$ and $C D$ would be diagonally opposite.

The rule for writing this as a quotient would be to start with $\mathbf{A}$ and go round the square counterclockwise, or widersinns, and write the quotient in the order indicated by the successive steps :

$$
\frac{\mathrm{AC}}{\overline{\mathrm{CB}}}, \frac{\mathrm{AC}}{\mathrm{CB}} \cdot \frac{\mathrm{BD}}{,}, \frac{\mathrm{AC}}{\overline{\mathrm{CB}}} \cdot \frac{\mathrm{BD}}{\overline{\mathrm{DA}}},
$$

which is very easily remembered.

The rule for changing from the square array to Cremona's linear array may be indicated by the symbol $X$ where the thick line denotes the diagonal first traversed. 
We may note that (i) if we interchange lines or columns or both the anharmonic ratio is unchanged thus :

$$
\left\{\begin{array}{c}
A D \\
C B
\end{array}\right\}=\left\{\begin{array}{c}
C B \\
A D
\end{array}\right\}=\left\{\begin{array}{l}
D A \\
B C
\end{array}\right\}=\left\{\begin{array}{l}
B C \\
D A
\end{array}\right\}=\lambda \text {. }
$$

This may be indicated also as follows:

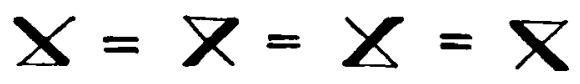

when we may interpret the symbol either to indicate the order in which the letters $A B C D$ are to be placed in square array to be read as above, or the order in which the letters in $\left\{\begin{array}{l}A D \\ C B\end{array}\right\}$ are to be read to give Cremona's linear order.

(ii) If we interchange the elements of either diagonal, and then apply (i) we get four anharmonic ratios indicated by
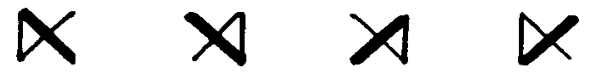

each being $=1 / \lambda$.

(iii) If we interchange the elements of either row, and then apply (i) we get
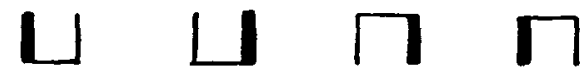

each being $=1-\lambda$.

(iv) If we interchange the elements of either column, and then apply (i) we get
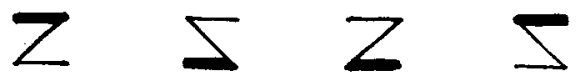

each being $=\lambda /(\lambda-1)$.

(v) If we keep one element fixed and interchange the others by one step cyclically round their triangle so that the element in the same column with the fixed one moves diagonally, or if we keep $A$ fixed and interchange the others by one clockwise cyclic step round their triangle and then apply (i) we get
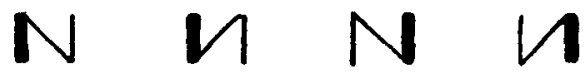

each being $=(\lambda-1) / \lambda$. 
(vi) If we proceed as in (v), except that "row" takes the place of "column" in the former part of the rule, and "counterclockwise" takes the place of "clockwise" in the latter part, we get

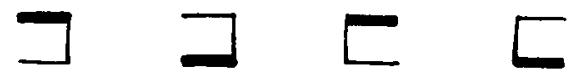

each being $=1 /(1-\lambda)$.

This exhausts the 24 possible orders, and verifies the fact that there are only 6 different values.

The group of operations by which we change from one to another of the orders of four points is obviously finite, as the number of such changes is ${ }^{24} \mathrm{P}_{2}=24 \times 23=552$.

But, algebraically, taking account only of the value of the anharmonic ratios, these operations are not all different. Indeed, as there are only 6 different values, the number of operations cannot be greater than ${ }^{6} \mathrm{P}_{2}=30$, and in fact they are only 5 in number, if we exclude the identical operation.

If by $\rho$ and $\phi$ we denote the operations thus defined: $\rho(\lambda) \equiv \frac{1}{\lambda}, \quad \phi(\lambda)=(1-\lambda)$; then $\rho \rho(\lambda)=\lambda$ or $\rho^{2}=1, \quad \therefore \rho=\rho^{-1}$. Similarly $\phi^{2}=1$ or $\phi=\phi^{-1}$.

Again $\phi \rho(\lambda)=(\lambda-1) / \lambda, \quad \rho \phi \rho(\lambda)=\lambda /(\lambda-1), \quad \phi \rho \phi \rho(\lambda)=\frac{1}{1-\lambda}$ $\rho \phi \rho \phi \rho(\lambda)=1-\lambda, \quad \phi \rho \phi \rho \phi \rho(\lambda)=\lambda$.

Here we take $\phi \rho(\lambda)$ to mean $\phi\{\rho(\lambda)\}$.

Thus all 6 values of the anharmonic ratio are got from $\lambda$ by combinations of the simple operations $\rho$ and $\phi$.

And from these results alone we could prove again that no other value of the anharmonic ratio can be reached by any combination of the operations $\rho$ and $\phi$. For since $\rho^{2}=1$ it follows that $\rho^{n}=\rho$ or 1 , according as $n$ is odd or even, and a similar rule holds for powers of $\phi$.

Hence the most general combination $\rho^{\alpha} \phi^{\beta} \rho^{\gamma} \ldots$ is reducible to $\rho \phi \rho . .$. , which again reduces to a product of alternate $\rho$ 's and $\phi$ 's not exceeding 5 factors, since $\rho \phi \rho \phi \rho \phi$ and $\phi \rho \phi \rho \phi \rho$ are each $=1$. If we abbreviate $\rho \phi \rho$ into the symbol $\psi$, we have

$$
\rho \phi \rho(\lambda)=\phi \rho \phi(\lambda)=\psi(\lambda)=\frac{\lambda}{\lambda-1} .
$$


We note that $(\phi \rho)^{3}$ and $(\rho \phi)^{3}$ are each $=1$, while $(\rho \phi \rho)^{2}=(\phi \rho \phi)^{2}=1$. Thus $\rho \phi \rho$ or $\phi \rho \phi$ is an operation similar to $\rho$ and $\phi$, inasmuch as the periodicity is 2 in each case, while the periodicities of the operators $a \equiv \rho \phi$ and $a^{-1} \equiv \phi \rho$ are each 3 .

The nature of the group of operations in question may be exhibited further by means of a diagram, by marking each line with the symbol for the operator by which the ratio at one of its ends is changed into that at the other end. Since all the simple operations excepting $a$ are reciprocal in character, the direction of the operation does not need to be specified. But in connection with $\alpha$ we shall introduce an arrowhead to be understood thus :

$$
\lambda-a \rightarrow-\mu \text { means } \mu=a(\lambda) \text {. }
$$

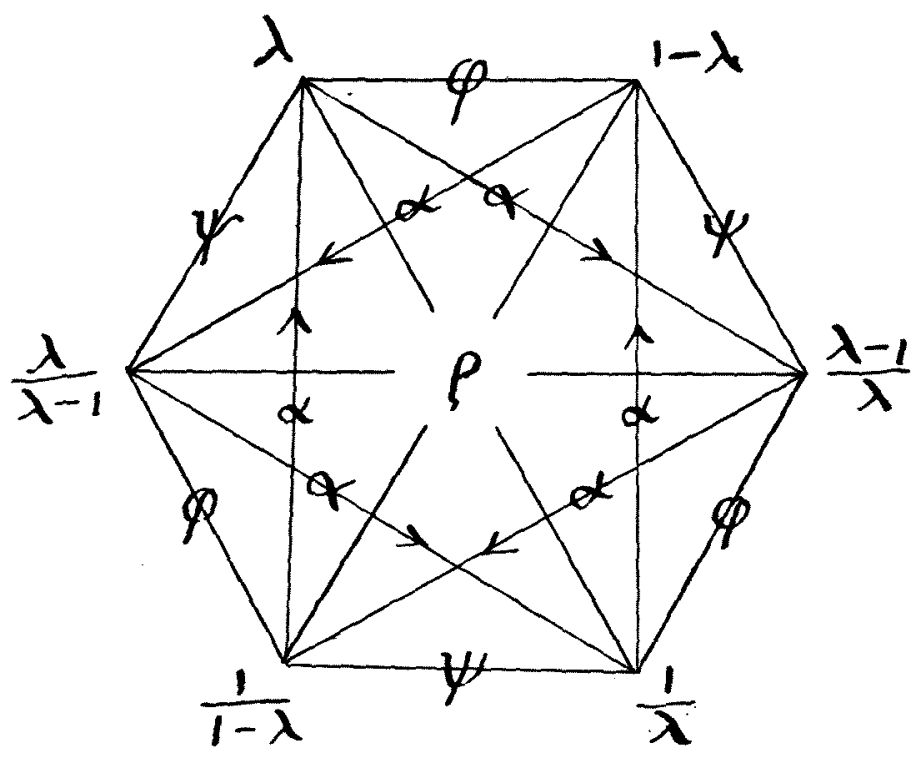

Fig. 3.

In this figure we see at a glance many properties of the operators.

For example, in the triangles whose sides are $\alpha \psi \phi$ the symbols occur invariably in that order, if we follow the direction of the 
arrowhead. This means, of course, that $a \psi \phi=1=\psi \phi a=\phi a \psi$. On the other hand $a^{-1} \phi \psi=1=\phi \psi a^{-1}=\psi a^{-1} \phi$, as is seen by going round these triangles in the opposite direction.

Note also the equilateral triangles giving $a \alpha a=1$. In fact, associating the symbols in any closed circuit in order, the combination $=1$ if we take $\alpha^{-1}$ instead of $\alpha$ when we are going opposite to the arrowhead. We see also $\phi \psi=\alpha, \psi \phi=\alpha^{-1}, \rho \psi=\alpha^{-1}$, etc.

This figure gives an apparently unique position to $\rho$ as compared with $\phi$ and $\psi$, but this does not imply a correspondingly unique property of the operator. In fact we could, by re-arranging the ratios round the hexagon, make $\rho$ and $\psi$ or $\rho$ and $\phi$ exchange places, without destroying the symmetry of the figure.

The group of six operations $1, \rho, \phi, \psi, a, a^{-1}$ is of course well known, being one of the simplest examples of the theory of groups, It has four sub-groups, besides the identical operation, viz. $1, \rho ; 1, \phi ; 1, \psi ; 1, a, a^{-1}$.

It may be proper to mention that the convention here used as to the order of writing a "product" of operators is the opposite to that employed in Burnside's standard treatise on the Theory of Groups of Finite Order.

In the particular case when $\lambda=-1$, the diagram of Fig. 1 reduces to

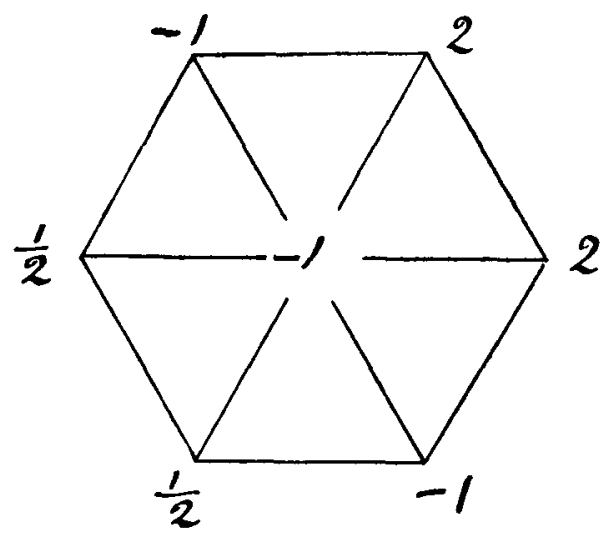

Fig. 4.

In this case there are but three different values of the anharmonic ratio to be got by altering the order of the points, and, 
using the marks explained above, we find that if $(A B C D)=-1$, the "Cremona" order to get the value -1 from the array $\begin{gathered}A C \\ D B\end{gathered}$ is indicated by any of the eight marks :-

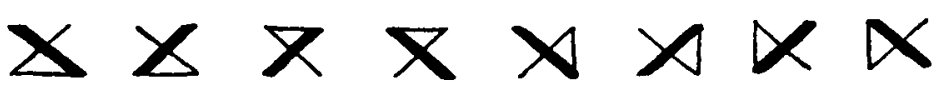

while the following set of eight:-

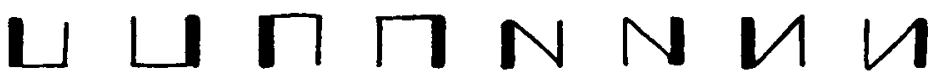

give the value 2 , and the following :-

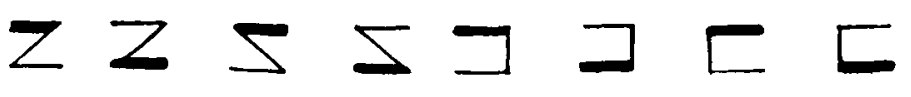

give the value $\frac{1}{2}$.

We may note that each of the first set of marks has two diagonal strokes; each of the second, two vertical strokes; and each of the third, two horizontal strokes.

Note.-The preceding notes were drawn up over thirteen years ago. Most of the information they convey is in one form or another well known, and is to be found in many Continental text books; but as it is not given in any one English book that I am acquainted with, I have thought some members of the Society may find the notes useful. 